Original Article

\title{
Fibroblast growth factor receptor 2 (FGFR2) fusions in Japanese patients with intrahepatic cholangiocarcinoma
}

\author{
Masanori Tsujie ${ }^{1}$ 1,*, Tomohisa Iwai ${ }^{2}$, Shoji Kubo ${ }^{3}$, Takashi Ura ${ }^{4}$, \\ Etsuro Hatano ${ }^{5}$, Daisuke Sakai ${ }^{6}$, Yutaka Takeda ${ }^{7}$, Masaki Kaibori ${ }^{8}$, \\ Tomoe Kobayashi $^{9}$, Akio Katanuma ${ }^{10}$, Yu Katayose ${ }^{11}$, Koji Fukase ${ }^{12}$, \\ Naoki Sakurai ${ }^{13}$, Yukiko Ito $^{14}$, Fumiya Sato ${ }^{15}$, Atsuyuki Maeda ${ }^{16}$, \\ Masanori Asada ${ }^{17}$, and Chigusa Morizane ${ }^{18}$
}

\begin{abstract}
${ }^{1}$ Department of Surgery, Osaka Rosai Hospital, Osaka, Japan, ${ }^{2}$ Department of Gastroenterology, Kitasato University Hospital, Sagamihara, Japan, ${ }^{3}$ Department of Hepato-Biliary-Pancreatic Surgery, Osaka City University Hospital, Osaka, ${ }^{4}$ Department of Clinical Oncology, National Hospital Organization Kyoto Medical Center, Kyoto, Japan, ${ }^{5}$ Department of Gastroenterological Surgery, Hyogo College of Medicine, Nishinomiya, Japan, ${ }^{6}$ Department of Gastroenterological Surgery, Osaka University Hospital, Suita, Japan, ${ }^{7}$ Department of Gastroenterological Surgery, Kansai Rosai Hospital, Amagasaki, Japan, ${ }^{8}$ Department of Surgery, Kansai Medical University, Osaka, Japan, ${ }^{9}$ Department of Gastroenterology and Hematology, Tomakomai City Hospital, Tomakomai, Japan, ${ }^{10}$ Center for Gastroenterology, Teine-Keijinkai Hospital, Sapporo, Japan, ${ }^{11}$ Department of Hepato-Biliary Pancreatic Surgery, Tohoku Medical and Pharmaceutical University Hospital, Sendai, Japan,, ${ }^{12}$ Department of Surgery, Japanese Red Cross Ishinomaki Hospital, Ishinomaki, Japan, ${ }^{13}$ Department of Surgery, Yamagata Prefectural Central Hospital, Yamagata, Japan, ${ }^{14}$ Department of Gastroenterology, Japanese Red Cross Medical Center, Tokyo, Japan, ${ }^{15}$ Department of Surgery, Kasugai Municipal Hospital, Kasugai, Japan, ${ }^{16}$ Department of Surgery, Ogaki Municipal Hospital, Ogaki, Japan, ${ }^{17}$ Department of Gastroenterology and Hepatology, Japanese Red Cross Osaka Hospital, Osaka, Japan and ${ }^{18}$ Department of Hepatobiliary and Pancreatic Oncology, National Cancer Center Hospital, Tokyo, Japan
\end{abstract}

*For reprints and all correspondence: Masanori Tsujie, Department of Surgery, Osaka Rosai Hospital, 1179-3

Nagasone-kitaku, Sakai, Osaka 591-8025, Japan. E-mail: tsujie@osakah.johas.go.jp

Received 16 November 2020; Editorial Decision 4 February 2021; Accepted 4 February 2021

\begin{abstract}
Objective: Fibroblast growth factor receptor gene alterations have emerged as promising drug targets for intrahepatic cholangiocarcinoma, a rare cancer that has a poor prognosis. This study evaluated the frequency of fibroblast growth factor receptor 2 fusions in clinical specimens from Japanese patients with iCCA.

Methods: This study enrolled 116 patients who had histologically or cytologically confirmed adenocarcinoma and been diagnosed as relapsing after resection or with unresectable intrahepatic cholangiocarcinoma. We evaluated the frequency of fibroblast growth factor receptor 2 fusionspositive cells in their specimens using break-apart fluorescent in situ hybridization 'for 114 patients who met the study protocol'.

Results: Of a total of 114 cases, six $(5.3 \%)$ were identified as fibroblast growth factor receptor 2 fusions-positive with a high frequency ( $87 \%$ or more) of fibroblast growth factor receptor 2 fusionspositive tumour cells whereas the remainder, with the exception of three cases with indeterminate
\end{abstract}


results, were identified as fibroblast growth factor receptor 2 fusions-negative. The patients' baseline characteristics as well as their objective response rates, disease control rates, times to progression, and times to treatment failure with previous or ongoing first-line chemotherapy did not have any obvious relationship to the proportion of fibroblast growth factor receptor 2 fusionspositive case.

Conclusions: Further detailed elucidation of fibroblast growth factor receptor 2 fusion status is expected to contribute to the development of promising therapeutic options for patients suffering from recurrent or unresectable intrahepatic cholangiocarcinoma.

Key words: FGFR2 fusions, intrahepatic cholangiocarcinoma, break-apart fluorescent in situ hybridization

\section{Introduction}

Fibroblast growth factor (FGF) signals through transmembrane tyrosine kinase receptors (FGFR1 to 4) to regulate angiogenesis, wound repair and many developmental pathways in the early embryo. FGF and FGFRs express in various types of human cells and play crucial roles in their proliferation, migration, differentiation and survival; thus aberrant FGFR signalling can promote tumour development $(1,2)$. In many cases of developmental disorder and cancer types, an association to the FGF/FGFR signalling pathway dysregulation has been reported (3). Examples of cancer types where genetic FGFR abnormalities are known are: gastric cancer (FGFR2 gene amplification incidence 10\%), breast cancer (FGFR1 and FGFR2 gene amplifications incidence $10 \%$ and $\sim 1 \%$, respectively), bladder cancer (FGFR3 activating mutation incidence $50-60 \%$ for the non-muscle invasive type), endometrial cancer (FGFR2 activating mutation incidence $12 \%$ ), and myeloma (FGFR3 translocation incidence $15 \%$ ) (1). It has also been reported that FGFR2 fusions by chromosomal translocation are found in $10-14 \%$ of intrahepatic cholangiocarcinoma (iCCA) $(4,5)$.

iCCA accounts for $10-20 \%$ of newly diagnosed liver cancers (6). Incidence and mortality rates for iCCA have risen over the last 15 years (7), and the prognosis for such patients is poor. In patients with unresectable disease or who relapse after surgery, standard first-line treatment with gemcitabine-cisplatin results in a median survival of $\sim 1.5$ years $(8-11)$, highlighting the need for more effective systemic therapies $(6,12)$. Recently, it has been reported that FGFR aberrations may be prognostic and may provide improved outcomes for patients treated with FGFR-targeted therapy (13). Several smallmolecule FGFR inhibitors are being investigated across multiple tumour types with FGFR alterations, including iCCA (14). Recently, pemigatinib, a selective inhibitor of FGFR1, 2 and 3, achieved a significant response in a phase 2 study (15) and was approved for iCCA in the USA. In addition, futibatinib, a selective inhibitor of FGFR1-4, resulted in a $34.3 \%$ objective response rate with interim data in iCCA patients harbouring FGFR2 gene fusions in a phase 2 study (16). Given this context, clarifying the frequency of FGFR2 gene abnormalities in iCCA is expected to contribute greatly to the development of novel FGFR2 kinase inhibitors as a therapeutic option. Thus, the aim of our study was to find the frequency of FGFR2 fusions in clinical specimens from Japanese patients with recurrent or unresectable iCCA.

\section{Patients and methods}

\section{Study design}

This study was a multicenter observational study. The aim of this study was to determine the frequency of FGFR2 fusions in clinical specimens from Japanese patients with recurrent or unresectable iCCA.
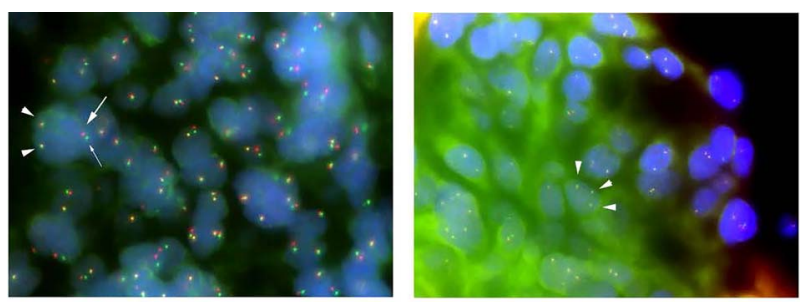

Figure 1. Representative fluorescence images for clinical cholangiocarcinoma spicemens stained with FGFR2 FISH probes in this study. In FGFR2 fusionspositive cells (left panel), green signal fluorescence (thin dotted arrow; indicates the FGFR2gene centromere region) was observed to stand apart from red signals (thick solid line arrow; indicates the FGFR2 gene telomere region). In the FGFR2 fusions-negative cells (right panel), only yellow signals (arrow heads; indicates a mixed fluorescence of red and green signals) were observed.

\section{Study population}

This study included 116 patients who were diagnosed with adenocarcinoma confirmed by histological or cytological findings and who were diagnosed by imaging to have either iCCA that was recurrent after curative resection for a primary tumour, or unresectable. Other criteria for eligibility were as follows: (i) first-line chemotherapy for the patient was planned, ongoing or complete, (ii) age $\geq 20$ years at written consent, (iii) Eastern Cooperative Oncology Group Performance Status score of 0 or 1 and (iv) clinical cholangiocarcinoma specimens 'from a primary lesion or metastatic lesions' at diagnosis or surgical resection were available. Any patients whom the investigator determined ineligible for this study were excluded.

The World Medical Association Declaration of Helsinki on medical research protocols and ethics was followed throughout the study. Authorization for the use of the clinical specimens for research purposes was obtained from the institutional review board of each study location.

\section{Break-apart fluorescent in situ hybridization}

To identify the presence of FGFR2 fusions, we used break-apart fluorescent in situ hybridization (FISH) methods, and $4 \mu \mathrm{m}$ sections from formalin-fixed, paraffin-embedded tumour specimens (4). More specifically, two kinds of human FGFR2 break-apart gene probes, FGFR2 telo (RP11-78A18 recognizing the FGFR2 gene telomere region) and FGFR2 cen (RP11-7P17 recognizing the FGFR2 gene centromere region), fluorescently labelled by nick translations in green and red, respectively, were purchased from Chromosome Science Labo (Sapporo, Japan). After hybridization, single sets of 100 tumour cells, each such tumour specimen being evaluated by two independent observers, were classified as FGFR2 fusions-positive or -negative under fluorescent microscopy (Fig. 1). In the event $7 \%$ or more FGFR2 fusions-positive cells were observed 
116 patients enrolled (FAS)

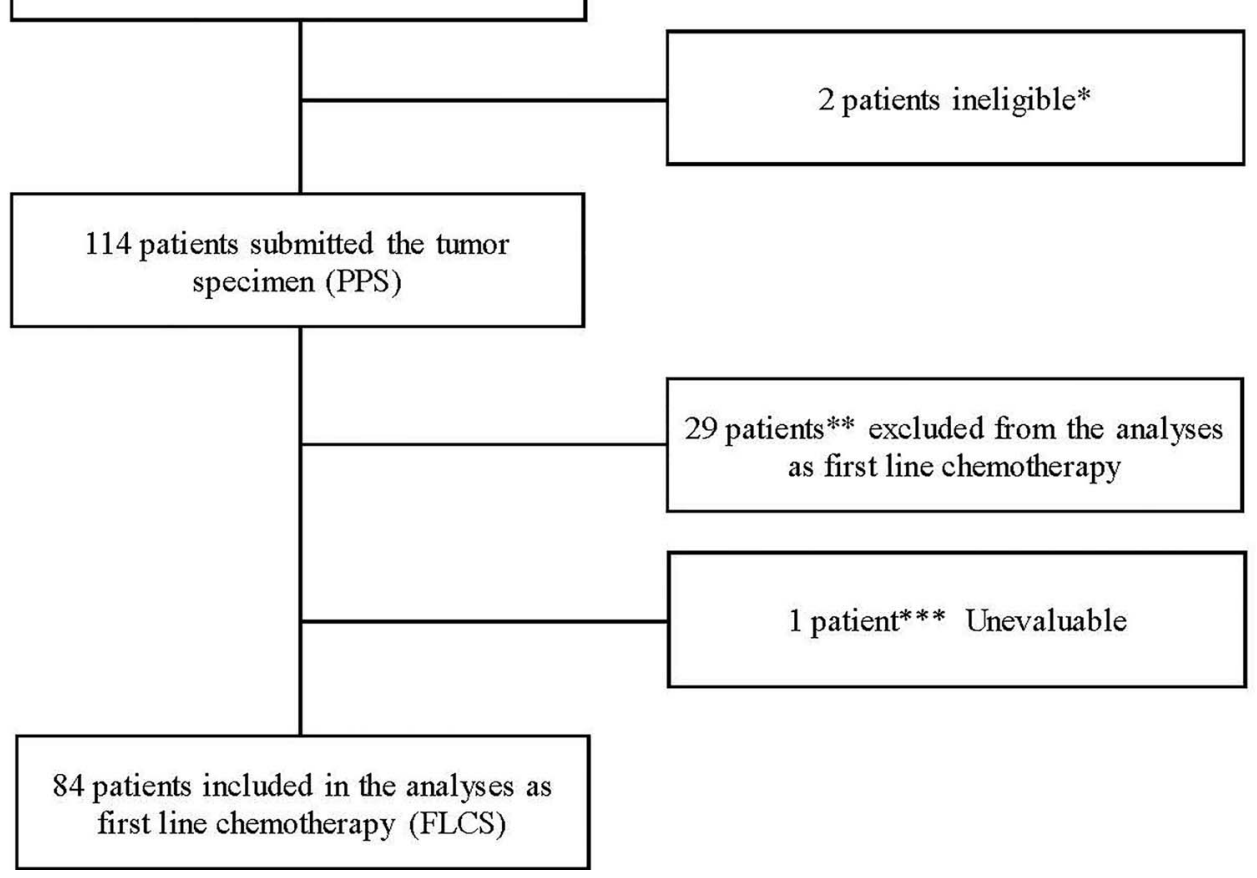

Figure 2. Disposition of cases FAS, full analysis set; PPS, per protocol set; FLCS, first-line chemotherapy set. *Deviation from inclusion criteria no. 1: Patient has histologically or cytologically confirmed adenocarcinoma and been diagnosed as relapsing or with unresectable intrahepatic cholangiocarcinoma by imaging. Patients histologically confirmed as bile duct cell carcinoma were enrolled in this trial. These patients were excluded from the PPS. **Six patients received first-line chemotherapy after giving informed consent from. Twenty-three patients did not receive first-line chemotherapy. These patients were excluded from FLCS. ${ }^{* * *}$ One patient could not be analysed for time to progression and time to treatment failure. Because the start and end date of first-line chemotherapy were unknown, this patient was excluded from FLCS.

in a tumour specimen, it was classed as FGFR2 fusions-positive (a positive case), and otherwise it was classed as FGFR2 fusionsnegative (a negative case). This cut-off value for the FISH analysis, was derived from our previous work on FGFR2 fusion-positive and -negative cases from surgically resected specimens. Fusion-negative cases validated by RNA sequencing showed background FISH signals at $6.4 \%$ (mean +2 standard deviations) (17). From the results, we initially defined the cut-off value of $\geq 7 \%$ for the positive cells in the FISH analysis.

\section{Statistical analysis}

Statistical significances in baseline characteristic distributions according to FGFR2 fusions status were analysed by Fisher's exact test with a $P<0.05$ two-side significance level. Age, gender, smoking history, alcohol consumption history, hepatitis $\mathrm{B}$ virus infection history and hepatitis $\mathrm{C}$ virus infection history were tested in these analyses. Where a first-line chemotherapy had been established, $95 \%$ confidence intervals for response rates and chemotherapy disease control rates were also examined according to FGFR2 fusions status. In addition, Kaplan-Meier Plots for time to progression and time to treatment failure with first-line chemotherapy were generated according to FGFR2 fusions status, and significance between the plots was analysed using Log-rank tests.

\section{Results}

\section{Disposition of cases}

A total of 116 cases were recruited maximally during the enrollment period from 2 October 2018 to 30 September 2019 (defined as the full analysis set: FAS). Two cases were excluded by reason of inclusion criteria violation, and the remaining 114 cases (defined as the per protocol set: PPS) comprised the $72(63.2 \%)$ males and $42(36.8 \%)$ females who were examined. The mean \pm standard deviation for age was $68.0 \pm 10.0$ years (range 37-84 years). At diagnosis, the primary tumour location for all the cases was determined the intrahepatic bile duct. Tumour specimens from 98 cases $(86.0 \%)$ were from a primary lesion and the remaining $16(14.0 \%)$ were from liver or other metastatic sites. The most frequent histological classifications were moderately differentiated adenocarcinoma (49 cases, $43.0 \%$ ) followed by poorly differentiated adenocarcinoma ( 29 cases, $25.4 \%$ ) and well differentiated adenocarcinoma (15 cases, $13.2 \%)$. These specimens were carried forward to histologically examine the frequency of FGFR2 fusions. Within the PPS, 84 cases for whom firstline chemotherapy was ongoing or complete prior to study inclusion, and who had definitive data on the start and end date of their chemotherapy were defined as the first-line chemotherapy set (FLCS) (Fig. 2).

\section{Frequency of FGFR2 fusions}

In this study, we focused on results from the 114 cases in the PPS (Fig. 2). FISH evaluation of FGFR2 fusion status identified six cases $(5.3 \%)$ with an FGFR2 fusions-positive tumour specimen, with high frequencies of $87 \%, 92 \%, 93 \%, 94 \%, 96 \%$ and $97 \%$ FISH-positive cells, respectively (Fig. 3). With exception of the three unevaluable (indeterminate) cases, all others were identified as FGFR2 fusionsnegative. Results on FGFR2 fusions status in the FLCS were similar to those in the PPS (Table 1). 
Table 1. Disposition of cases according to FGFR2 fusions status

\begin{tabular}{lllll}
\hline Analysis Sets & \multicolumn{2}{l}{ FGFR2 fusions } & Total & \\
\cline { 2 - 3 } & Positive $(n=6)$ & Negative $(n=107)$ & Indeterminate $(n=3)$ & 116 \\
\hline All enrolled patients & 6 & 107 & 3 & $116(100.0 \%)$ \\
FAS & $6(100.0 \%)$ & $107(100.0 \%)$ & $3(100.0 \%)$ & $0(0.0 \%)$ \\
Patients Eexcluded from FAS & $0(0.0 \%)$ & $0(0.0 \%)$ & $0(0.0 \%)$ & $114(98.3 \%)$ \\
PPS & $6(100.0 \%)$ & $105(98.1 \%)$ & $3(100.0 \%)$ & $2(1.7 \%)$ \\
Patients excluded from PPS & $0(0.0 \%)$ & $2(1.9 \%)$ & $0(0.0 \%)$ & $84(73.7 \%)$ \\
FLCS & $4(66.7 \%)$ & $77(73.3 \%)$ & $3(100.0 \%)$ & $30(26.3 \%)$ \\
Patients excluded from FLCS & $2(33.3 \%)$ & $28(26.7 \%)$ & $0(0.0 \%)$ & \\
\hline
\end{tabular}

Analysis set: All enrolled patients.

FAS, full analysis set; PPS, per protocol set; FLCS, first-line chemotherapy set

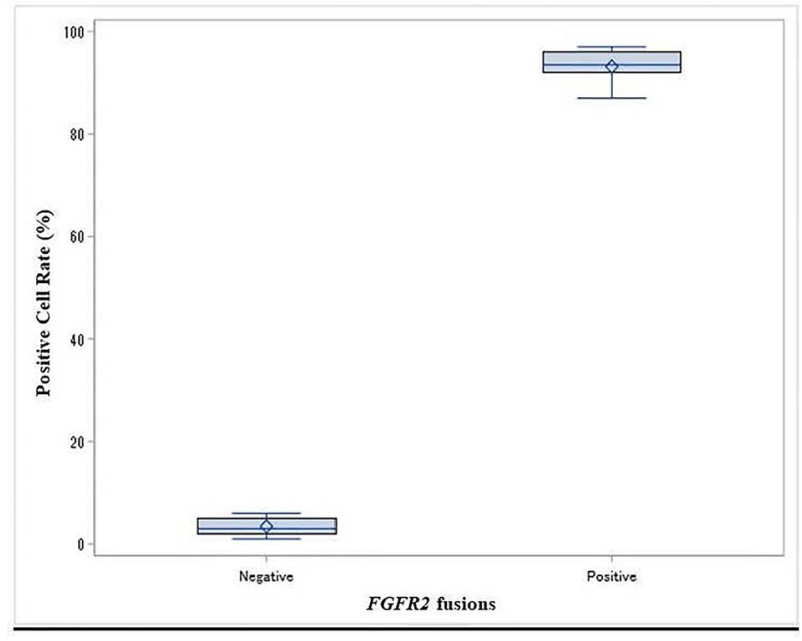

Figure 3. FGFR2 fusions-positive cell rates in tum or specimens from patients with intrahepatic cholangiocarcinoma. Each column represents the box plot of FGFR2 fusions-negative $(n=107)$ and positive $(n=6)$ cases composed of the minimum value, the lower quartile, the median, the mean value (white diamond), the upper quartile and the maximum value. The individual frequency of the FGFR2 fusions-positive cases were $87 \%, 92 \%, 93 \%, 94 \% .96 \%$ and $97 \%$, respectively.

The total success rate of the FISH assay was 97.4\% (113/116), including $94.0 \%(109 / 116)$ succeeded in the first evaluations. A median turnaround time (TAT) was 18 days (range 13-27 days), which was results from 116 cases of FAS. TAT was calculated as the difference in calendar days between the date the specimen was submitted by the site to the date of the report for the specimens.

\section{Baseline characteristics according to the FGFR2 fusions status}

Baseline characteristics for the PPS: mean age \pm standard deviation was $64.7 \pm 7.4$ years (range 52-73 years) for FGFR2 fusions-positive cases (positive cases, $n=6$ ) and 68.2 \pm 10.1 years (range 3784 years) for FGFR2 fusions-negative cases (negative cases, $n=105$ ), respectively. Gender for all positive cases was male whereas the negative group was composed of 63 males $(60.0 \%)$ and 42 females $(40.0 \%)$. As for smoking, all positive cases had a smoking history, whereas there were 60 cases $(57.1 \%)$ with smoking history among the negative cases. Alcohol consumption history was noted in five $(83.3 \%)$ of the positive cases and $56(53.3 \%)$ of the negative. History of hepatitis B virus infection was reported in three $(50.0 \%)$ of the positive cases and $28(26.7 \%)$ of the negative. Hepatitis C virus infection history was not reported in any positive cases whereas six $(5.7 \%)$ of the negative cases did report this history (Table 2). Thus maleness, smoking history, alcohol consumption history and hepatitis $B$ virus infection history proportions were higher in the positive cases than in the negative. In addition, the proportion of hepatitis $\mathrm{C}$ virus infection histories in the positive cases was lower than that in the negative. Nevertheless, we found no statistically significant differences, by way of age, gender, smoking history, alcohol consumption history, hepatitis $B$ virus infection history or hepatitis $C$ virus infection history $(P>0.05$, Fisher's exact test $)$.

\section{First-line chemotherapy prior to inclusion according to the FGFR2 fusions status}

The 84-case of FLCS was composed of 45 recurrent cases $(53.6 \%)$, being recurrent after curative resection for primary tumours, and 39 who were unresectable $(46.4 \%)$. The most frequent chemotherapy regimen was gemcitabine plus cisplatin for 57 cases $(67.9 \%)$ followed by S-1 monotherapy for 15 (17.9\%), S-1 plus gemcitabine plus cisplatin for six $(7.1 \%)$ and gemcitabine monotherapy for six cases (7.1\%).

Response to these chemotherapy regimens is summarized in Table 3. The response rates in the FGFR2 fusions-positive and negative cases were $0 \%$ (95\% confidence interval: $0.0-49.0 \%$ ) and $14.3 \%$ (95\% confidence interval: $8.2-23.8 \%$ ), respectively. Because of the overlapping $95 \%$ confidence intervals, no difference could be declared between the positive and negative cases. In addition, Kaplan-Meier Plots of both time to progression and time to chemotherapy treatment failure (data not shown) revealed no statistical difference between the positive and negative cases $(P=0.5357$ and $P=0.9783$, respectively, Log-rank test $)$.

\section{Discussion}

FGFR2 tyrosine kinase fusions have been identified as novel oncogenic (3) and druggable targets (13) in various cancers, especially in iCCA. Thus, the aim of this multicenter observational study was to elucidate FGFR2 fusion frequency using FISH methods, as well as reliable baseline factors, in Japanese patients with recurrent or unresectable iCCA.

The results of our study revealed that 6 out of the 114 cases examined were identifiable as FGFR2 fusions-positive. In addition, the individual proportion of FGFR2 fusions-positive cells counted in the FISH-labelled tumour specimens was as high as $87 \%$ or more in these cases. Although the role of FGFR2 fusions in the pathogenesis 
Table 2. Baseline characteristics according to FGFR2 fusions status

\begin{tabular}{|c|c|c|c|c|c|}
\hline \multirow[t]{2}{*}{ Item } & \multirow[t]{2}{*}{ Category } & \multicolumn{3}{|l|}{ FGFR2 fusions } & \multirow[t]{2}{*}{ Total } \\
\hline & & Positive $(n=6)$ & Negative $(n=105)$ & Indeterminate $(n=3)$ & \\
\hline \multirow[t]{2}{*}{ Age } & $<65$ & $3(50.0 \%)$ & $32(30.5 \%)$ & $1(33.3 \%)$ & $36(31.6 \%)$ \\
\hline & $\geqq 65$ & $3(50.0 \%)$ & $73(69.5 \%)$ & $2(66.7 \%)$ & $78(68.4 \%)$ \\
\hline \multirow[t]{2}{*}{ Gender } & Male & $6(100.0 \%)$ & $63(60.0 \%)$ & $3(100.0 \%)$ & $72(63.2 \%)$ \\
\hline & Female & $0(0.0 \%)$ & $42(40.0 \%)$ & $0(0.0 \%)$ & $42(36.8 \%)$ \\
\hline \multirow[t]{2}{*}{ Smoking history } & Current or former & $6(100.0 \%)$ & $60(57.1 \%)$ & $2(66.7 \%)$ & $68(59.6 \%)$ \\
\hline & Never & $0(0.0 \%)$ & $45(42.9 \%)$ & $1(33.3 \%)$ & $46(40.4 \%)$ \\
\hline \multirow[t]{2}{*}{ Drinking history } & Current or former & $5(83.3 \%)$ & $56(53.3 \%)$ & $3(100.0 \%)$ & $64(56.1 \%)$ \\
\hline & Never & $1(16.7 \%)$ & $49(46.7 \%)$ & $0(0.0 \%)$ & $50(43.9 \%)$ \\
\hline \multirow[t]{2}{*}{ Hepatitis B virus infection history } & None & $3(50.0 \%)$ & $77(73.3 \%)$ & $3(100.0 \%)$ & $83(72.8 \%)$ \\
\hline & Yes & $3(50.0 \%)$ & $28(26.7 \%)$ & $0(0.0 \%)$ & $31(27.2 \%)$ \\
\hline \multirow[t]{2}{*}{ Hepatitis $\mathrm{C}$ virus infection history } & None & $6(100.0 \%)$ & $99(94.3 \%)$ & $3(100.0 \%)$ & $108(94.7 \%)$ \\
\hline & Yes & $0(0.0 \%)$ & $6(5.7 \%)$ & $0(0.0 \%)$ & $6(5.3 \%)$ \\
\hline
\end{tabular}

Analysis set: PPS.

Table 3. Response to first-line chemotherapy prior to inclusion according to FGFR2 fusions status

\begin{tabular}{|c|c|c|c|c|}
\hline \multirow[t]{2}{*}{ Responses } & \multicolumn{3}{|l|}{ FGFR2 fusions } & \multirow[t]{2}{*}{ Total } \\
\hline & Positive $(n=4)$ & Negative $(n=77)$ & Indeterminate $(n=3)$ & \\
\hline Complete response (CR) & $0(0.0 \%)$ & $0(0.0 \%)$ & $0(0.0 \%)$ & $0(0.0 \%)$ \\
\hline Partial response (PR) & $0(0.0 \%)$ & $11(14.3 \%)$ & $1(33.3 \%)$ & $12(14.3 \%)$ \\
\hline Stable disease (SD) & $1(25.0 \%)$ & $25(32.5 \%)$ & $1(33.3 \%)$ & $27(32.1 \%)$ \\
\hline Non-CR/Non-PD & $0(0.0 \%)$ & $1(1.3 \%)$ & $0(0.0 \%)$ & $1(1.2 \%)$ \\
\hline Progressive disease (PD) & $1(25.0 \%)$ & $14(18.2 \%)$ & $0(0.0 \%)$ & $15(17.9 \%)$ \\
\hline Not evaluable (NE) & $2(50.0 \%)$ & $26(33.8 \%)$ & $1(33.3 \%)$ & $29(34.5 \%)$ \\
\hline Response rate $(\mathrm{CR}+\mathrm{PR})$ & $0(0.0 \%)$ & $11(14.3 \%)$ & $1(33.3 \%)$ & $12(14.3 \%)$ \\
\hline $95 \%$ confidence interval $(\%)$ & {$[0.0,49.0]$} & {$[8.2,23.8]$} & {$[6.1,79.2]$} & {$[8.4,23.3]$} \\
\hline Disease control rate & $1(25.0 \%)$ & $37(48.1 \%)$ & $2(66.7 \%)$ & $40(47.6 \%)$ \\
\hline$(\mathrm{CR}+\mathrm{PR}+\mathrm{SD}+$ Non-CR/Non-PD $)$ & & & & \\
\hline $95 \%$ confidence interval $(\%)$ & {$[4.6,69.9]$} & {$[37.3,59.0]$} & {$[20.8,93.9]$} & {$[37.3,58.2]$} \\
\hline
\end{tabular}

Analysis set: FLCS.

of cholangiocarcinoma is still being actively investigated, FGFR2 fusions are already thought to drive oncogenesis in cholangiocarcinoma (18). The high frequency of FGFR2 fusions-positive cells found in a subset of this study suggest that enhanced tumour cell proliferation driven by the presence on FGFR2 fusions was the main contributor to tumour progression in those. As for the percentage of our FGFR2 fusions-positive cases, previous genomic analysis studies that analysed the surgically resected cases have reported that FGFR2 fusions are implicated in $13-14 \%$ of patients with iCCA $(4,5)$. In contrast, the present study with recurrent or unresectable iCCA and recent reports $(15,19)$ that targeted advanced/recurrent patients indicated lower frequency $(5.3 \%$ and $7.4-9 \%$, respectively). Recently, it was reported that FGFR2 fusions were related to better overall survival $(12,20)$. In addition, it was reported that the activation of the FGF/FGFR pathway associates with better prognosis in patients with iCCA (21). Low frequency of FGFR2 fusions in the patients with recurrent or unresectable iCCA in the present study may be related to this point. However, the percentage of FGFR2 fusions-positive cases found in this study was less than in previous reports. Our lower percentage of positive cases may be explained by our study's relatively small sample size, which may have skewed our results due to a large deviation in the proportion of positive cases, although we did reaffirm that the considerable percentage of FGFR2 gene abnormalities for patients suffering from recurrent or unresectable iCCA was present.

Previously, Kuwata et al. reported a next-generation sequencing (NGS) success rate of $82.9 \%$ (22). In this study, we observed a FISH success rate $\sim 14 \%$ higher than their NGS success rate. On the other hand, our median TAT was 18 days longer than that previous reported with the NGS (23) because of limitations whereby we had to do our analyses after pooling samples from multiple centers in this study. If we had had no analysis time restrictions, our TAT would have been shorter. We therefore posit that FISH analyses may have more clinical utility, since physicians and patients deserve the most accurate diagnostic test results, in the shortest possible TAT.

We also analysed baseline characteristics and results for first-line chemotherapy prior to study inclusion to look for factors presumably due to the presence of an FGFR2 fusions-positive tumour. Here, the proportions of maleness, smoking history, alcohol consumption history and hepatitis B virus infection history were higher in our FGFR2 fusions-positive cases than in our negative. In contrast, the proportion of hepatitis $\mathrm{C}$ virus infection histories in our positive cases was lower than that in our negative. However, no statistical significance was noted for any of these factors. In addition, there were 
no significant differences between our FGFR2 fusions-positive and negative cases due to first-line chemotherapy prior to study inclusion.

It has been reported that FGFR2 fusions are associated with female sex $(5,24)$ and young age of onset $(5,19,24)$. Furthermore, Arai et al. (4) reported no significant differences in age, gender, tumour differentiation, clinical stage and prognosis except for a propensity of hepatitis virus infections, between their fusion-positive and -negative cases. The results from our present study were similar to those reported. At the same time, our results imply that, unless one does chromosomal tumour examinations, the development of any screening algorithm comprised of several baseline characteristics, to find possible FGFR2 fusions-positive cases in an iCCA patient population, in whom to apply FGFR inhibitors in future, will likely be difficult.

Further detailed elucidation of patients' FGFR2 fusion status is expected to contribute to the development of promising therapeutic options for those suffering from iCCA.

\section{Conclusion}

The present multicenter observational study has elucidated FGFR2 fusions status in a population of Japanese patients with recurrent or unresectable iCCA.

\section{Acknowledgements}

We gratefully acknowledge the following investigators who participated in this multicenter study: Michio Nakamura, MD (Sapporo City General Hospital, Sapporo, Japan), Masayoshi Dazai, MD (Sapporo Medical Center NTT EC, Sapporo), Susumu Sogabe, MD (KKR Sapporo Medical Center, Sapporo), Yasushi Tsuji, MD (KKR Sapporo Medical Center Tonan Hospital, Sapporo), Yasuo Takahashi, MD (Hokkaido Cancer Center, National Hospital Organization, Sapporo), Takuto Miyagishima, MD (Kushiro Rosai Hospital. Kushiro, Japan), Kazuteru Hatanaka, MD (Hakodate Municipal Hospital, Hakodate, Japan), Masaya Oikawa, MD (Sendai Open Hospital, Sendai), Hideaki Kodama, MD (Sendai Medical Center, Sendai), Hajime Iwasashi, MD (Miyagi Cancer Center, Natori, Japan), Shigeru Maruhashi, MD (Fukushima Medical University Hospital, Fukushima, Japan), Shinsuke Funakoshi, MD (Tokyo Saiseikai Central Hospital, Tokyo), Hideaki Motohashi, MD (Moriyama Memorial Hospital, Tokyo), Mitsuyoshi Honjyo, MD (Tokyo Medical University Hospital, Tokyo), Kazuya Sugimiri, MD (Yokohama City University Medical Center, Yokohama, Japan), Hidenobu Masui, MD (Yokosuka Kyosai Hospital, Yokosuka, Japan), Kazuya Koizumi, MD (Shonankamakura General Hospital, Kamakura, Japan), Eiji Sakamoto, MD (Japanese Red Cross Nagoya Daini Hospital, Nagoya, Japan), Toshiyuki Arai, MD (Anjo Kosei Hospital, Anjo, Japan), Akira Itou, MD (Toyohashi Municipal Hospital, Toyohashi, Japan), Daisuke Yamaguchi, MD (Kyoto Katsura Hospital, Kyoto), Kazuyoshi Matsumura, MD (Shiga General Hospital, Moriyama, Japan), and Hiroaki Terajima, MD (Tazuke Kofukai Medical Institute, Kitano Hospital, Osaka). This study is sponsored by Taiho Pharmaceutical Co., Ltd. Funding to pay the Open Access publication charges for this article was provided by Taiho Pharmaceutical Co., Ltd.

\section{Conflict of interest statement}

D. Sakai received lecture fee from Chugai Pharmaceutical Co. Ltd, Daiichi-Sankyo Co. Ltd and received grant from Chugai Pharmaceutical Co. Ltd, Daiichi-Sankyo Co. Ltd, Eli Lilly Japan K.K., Ono Pharmaceutical Co. Ltd, Yakult Honsha. E. Hatano received lecture fee and donation from Taiho Pharmaceutical Co., Ltd. C. Morizane received honoraria from Eisai, MSD K.K., Novartis, Taiho Pharmaceutical, Teijin Pharma, Yakult Honsha and received grant from AstraZeneca, Eisai, J-Pharma, Merck biopharma, ONO Pharmaceutical Co.,LTD, Taiho Pharmaceutical, Yakult Honsha and has advisory role Abbvie, AstraZeneca, J-Pharma, MSD K.K., Novartis, Taiho Pharmaceutical, Teijin Pharma, Yakult Honsha.

\section{References}

1. Turner N, Grose R. Fibroblast growth factor signaling: from development to cancer. Nat Rev Cancer 2010;10:116-29.

2. Liang G, Liu Z, Wu J, Cai Y, Li X. Anticancer molecules targeting fibroblast growth factor receptors. Trends Pharmacol Sci 2012;33:531-41.

3. Brooks AN, Kilgour E, Smith PD. Molecular pathways: fibroblast growth factor signaling: a new therapeutic opportunity in cancer. Clin Cancer Res 2012;18:1855-62.

4. Arai Y, Totoki Y, Hosoda F, et al. Fibroblast growth factor receptor 2 tyrosine kinase fusions define a unique molecular subtype of cholangiocarcinoma. Hepatology 2014;59:1427-34.

5. Graham RP, Barr Fritcher EG, Pestova E, et al. Fibroblast growth factor receptor 2 translocations in intrahepatic cholangiocarcinoma. Hum Pathol 2014;45:1630-8.

6. Massarweh NN, El-Serag HB. Epidemiology of hepatocellular carcinoma and intrahepatic cholangiocarcinoma. Cancer Control 2017;24:1073274817729245.

7. Saha SK, Zhu AX, Fuchs CS, Brooks GA. Forty-year trends in cholangiocarcinoma incidence in the U.S.: intrahepatic disease on the rise. Oncologist 2016;21:594-9.

8. Okusaka T, Nakachi K, Fukutomi A, et al. Gemcitabine alone or in combination with cisplatin in patients with biliary tract cancer: a comparative multicentre study in Japan. Br J Cancer 2010;103:469-74.

9. Valle J, Wasan H, Palmer DH, et al. Cisplatin plus gemcitabine versus gemcitabine for biliary tract cancer. N Engl J Med 2010;362:1273-81.

10. Morizane C, Okusaka T, Mizusawa J, et al. Combination gemcitabine plus $\mathrm{S}-1$ versus gemcitabine plus cisplatin for advanced/recurrent biliary tract cancer: the FUGA-BT (JCOG1113) randomized phase III clinical trial. Ann Oncol 2019;30:1950-8.

11. Lamarca A, Hubner RA, David Ryder W, Valle JW. Second-line chemotherapy in advanced biliary cancer: a systematic review. Ann Oncol 2014;25:2328-38.

12. Jain A, Borad MJ, Kelley RK, et al. Cholangiocarcinoma with FGFR genetic aberrations: a unique clinical phenotype. JCO Precision Oncology 2018;DOI 10.1200/PO.17.00080.

13. Dai S, Zhou Z, Chen Z, Xu G, Chen Y. Fibroblast growth factor receptors (FGFRs): structures and small molecule inhibitors. Cells 2019;8:614.

14. Lombardi P, Marino D, Fenocchio E, Chilà G, Aglietta M, Leone F. Emerging molecular target antagonists for the treatment of biliary tract cancer. Expert Opin Emerg Drugs 2018;23:63-75.

15. Abou-Alfa GK, Sahai V, Hollebecque A, et al. Pemigatinib for previously treated, locally advanced or metastatic cholangiocarcinoma: a multicentre, open-label. phase 2 study. Lancet Oncol 2020;21:671-84.

16. Goyal L, Meric-Bernstam F, Hollebecque A, et al. FOENIX-CCA2: A phase II, open-label, multicenter study of futibatinib in patients (pts) with intrahepatic cholangiocarcinoma (iCCA) harboring FGFR2 gene fusions or other rearrangements. ASCO Meeting Library 2020:Abstract 108. Available from: https://meetinglibrary.asco.org/record/186878/abstract.

17. Nakamura H, Arai Y, Totoki Y, et al. Genomic spectra of biliary tract cancer. Nat Genet 2015;47:1003-100.

18. Valle JW, Lamarca A, Goyal L, Barriuso J, Zhu AX. New horizons for precision medicine in biliary tract cancers. Cancer Discov 2017;7:943-62.

19. Maruki Y, Morizane C, Arai Y, et al. Molecular detection and clinicopathological characteristics of advanced/recurrent biliary tract carcinomas harboring the FGFR2 rearrangements: a prospective observational study (PRELUDE study). J Gastroenterol Epub 2020;26.

20. Churi CR, Shroff R, Wang Y, et al. Mutation profiling in cholangiocarcinoma: prognostic and therapeutic implications. PLoS One 2014;9:e115383. 
21. Javle M, Bekaii-Saab $\mathrm{T}$, Jain $\mathrm{A}$, et al. Biliary cancer: utility of next-generation sequencing for clinical management. Cancer 2016;122:3838-47.

22. Kuwata T, Wakabayashi M, Hatanaka Y, et al. Impact of DNA integrity on the success rate of tissue-based next-generation sequencing: lessons from nationwide cancer genome screening project SCRUM-Japan GISCREEN. Pathol Int 2020;; 70:932-42.
23. Weiss GJ, Hoff BR, Whitehead RP, Sangal A, et al. Evaluation and comparison of two commercially available targeted next-generation sequencing platforms to assist oncology decision making. Onco Targets Ther 2015;8:959-67.

24. Milind J, Tanios BS, Apurva J, et al. Biliary cancer: utility of next-generation sequencing for clinical management. Cancer 2016;122:3838-47. 